\title{
THE CRITICAL NEIGHBOURHOOD RANGE FOR ASYMPTOTIC OVERLAY CONNECTIVITY IN DENSE AD HOC NETWORKS
}

\author{
Sandrine Calomme and Guy Leduc \\ Research Unit in Networking \\ Electrical Engineering and Computer Science Department \\ University of Liège, Belgium \\ Email: $\{$ calomme,leduc\}@run.montefiore.ulg.ac.be
}

\begin{abstract}
We define, for an overlay built on top of an ad hoc network, a simple criterion for neighbourhood: two overlay nodes are neighbours if and only if there exists a path between them of at most $R$ hops, and $R$ is called the (overlay) neighbourhood range. A small $R$ may result in a disconnected overlay, while an unnecessarily large $R$ would generate extra control traffic. We are interested in the minimum $R$ ensuring overlay connectivity, the so-called critical $R$.

We derive a necessary and sufficient condition on $R$ to achieve asymptotic connectivity of the overlay almost surely, i.e. connectivity with probability 1 when the number of overlay nodes tends to infinity, under the hypothesis that the underlying ad hoc network is itself asymptotically almost surely connected.

This condition, though asymptotic, sheds some light on the relation linking the critical $R$ to the number of nodes $n$, the normalized radio transmission range $r$ and the overlay density $D$ (i.e., the proportion of overlay nodes). This condition can be considered as an approximation when the number of nodes is large enough. Since $r$ is considered as a function of $n$, we are able to study the impact of topology control mechanisms, by showing how the shape of this function impacts the critical $R$.
\end{abstract}

Keywords: ad hoc networks, overlay, connectivity, topology control

\section{Introduction}

In a previous work, we adopted an overlay approach for the introduction of the active technology in ad hoc networks [Calomme and Leduc, 2004]. The framework proposed allowed active nodes to inject cus- 
tomized routing protocols in the network to communicate all together, or to use any upper-layer active application, in order to improve the communication performance.

More generally, as most application of MANETs involve group communication [Mohapatra et al., 2004] and as grouping behaviour of the mobile users has been observed [Wang and Li, 2002; Tang and Baker, 2000], most wireless ad hoc networks can be seen as composed of one or several communities. The nodes of these communities can be characterized by a common specialized hardware, such as a sensor, or software, such as an active platform, or share a custom routing protocol or application. In all cases, they can use and take advantage of their common enhanced capabilities if and only if they are able to communicate efficiently through the other nodes, that is if and only if they are organized as an overlay.

Overlay advantages come however at the expense of the overlays creation, usage and maintenance, that must be kept moderate. Consequently, a full mesh is probably not the most adapted nor efficient solution for overlay applications. A natural rule of thumb is to admit as overlay neighbours a set of close overlay nodes, the distance measure employed being the number of hops. Two approaches are possible. One can fix the cardinality of the set of neighbours or the maximum number of hops admitted between overlay neighbours. We adopt the latter one. In this case, the maximum distance between two neighbours is an integer value that must be sufficiently high to obtain a connected overlay but as low as possible to limit the amount of messages generated in the network by overlay nodes communication.

The parallel with topology control in ad hoc networks is obvious. To achieve connectivity, each ad hoc node could use its maximum transmission range, in order to reach many neighbours. However, mobile devices have a limited amount of battery power. Moreover, this would create a lot of interferences, reducing the overall capacity of the network. With a homogeneous topology control algorithm, all nodes adopt the same transmitting range value. The critical transmitting range problem consists of determining the minimum value that generates a connected network. We have adopted a similar terminology for our problem: the maximum number of hops allowed between overlay neighbours is called the neighbourhood range and the determination of its best value the critical neighbourhood range problem.

This paper is structured as follows. In Sect. 2, we give an overview on previous related work over the critical transmission range. In Sect. 3 , we precisely define the problem studied. In Sect. 4, we present ana- 
lytical results and discuss some of their practical implications. We then conclude.

\section{Related Work}

In many realistic scenarios, node positions are not known in advance. Hence a probabilistic approach is used in every analytical study of the critical transmission range problem.

First studies of graph connectivity were developed in the context of the random graphs theory. A random graph is a graph generated by some random procedure [Bollobas, 1985]. In 1960, Erdos and Rényi [Erdos and Rényi, 1960] showed that for many monotone-increasing properties of random graphs, like connectivity, graphs of a size slightly less than a certain threshold are very unlikely to have the property, whereas graphs with a few more graph edges are almost certain to have it. This is known as a phase transition phenomenon.

In classical random graph models, there is no a priori structure. All vertices are equivalent and there is no correlation between different edges existence. In ad hoc and sensor networks, nodes are more likely to be direct neighbours if they are located close to each other. Therefore random geometric graphs are more suited to model them. Random geometric graphs are constructed by placing points at random according to some arbitrary specified density function on a $d$-dimensional Euclidean space and connecting nearby points [Penrose, 2003]. Some of the geometric random graphs results can be applied in the study of connectivity in ad hoc and sensor networks [Penrose, 1999]. Various transition phenomena can also be observed in geometric random graphs [Krishnamachari et al., 2001]. Monotone properties for this class of graphs have sharp treshold [Goel et al., 2004]. Asymptotically, as the network density tends to infinity, a critical value transmission range can be established [Gupta and Kumar, 1999].

We are not aware of any work related to the critical (overlay) neighbourhood range problem. In the following sections, we define it in more details, and we solve it using known results on the critical transmission range problem cited above.

\section{Problem Definition and Discussion}

We are interested in the asymptotic connectivity of overlay graphs built over asymptotically almost surely (a.a.s.) connected basic graphs.

These notions are defined in the following paragraphs. We then discuss the implicit assumptions we make in the problem and model specification. 


\section{Basic and Overlay Graphs}

Consider an ad hoc network of $n$ nodes, deployed over a square field of unitary area, and where each node is assigned a normalized transmission range of length $r$. This network is modelled by a random geometric graph denoted $g(n, r)$ which has the following properties.

The vertices of $g$ are uniformly and independently distributed on the unitary square. They can either have been disseminated following the uniform distribution of $n$ points or by a spatial homogeneous Poisson point process of mean $n$.

There exists an edge between each pair of vertices if and only if the Euclidean distance between them is not greater than $r$.

Let then $g(n, r)$ be a connected graph, $D$ be a real number with $0 \leq D \leq 1$ and $R$ be an integer with $R \geq 1$.

An overlay graph $G(n, r, D, R)$ denotes a graph with the following properties.

The $D$ parameter represents the overlay nodes density. The number of vertices of $G$ equals the lowest integer above a proportion $D$ of the number of vertices of $g$. These are randomly and uniformly selected in the vertices set of $g$, which is called its basic graph.

The parameter $R$ is called the neighbourhood range. There exists an edge between a pair of vertices $\left(v_{1}, v_{2}\right)$ if and only if the shortest path in $g$ from $v_{1}$ to $v_{2}$ contains less than or exactly $R$ hops.

In the following, in conjunction with the ad hoc and sensor networks terminology, the vertices of an overlay graph will be referred to as overlay nodes and the vertices of its basic graph as nodes.

\section{Asymptotic Connectivity}

Let all graph parameters be a function of the number of nodes. For example, $r(n)$ can be decreasing when $n$ increases, which is a desired behaviour for minimizing the capacity loss due to interferences.

A basic graph can be denoted by $g(n, r(n)$ and an overlay graph by $G(n, r(n), D(n), R(n))$ or $G(g, D(n), R(n))$. We may generally simply write $g(n, r), G(n, r, D, R)$ or, if $g(n, r)$ is given, $G(g, D, R)$.

DEFINITION 1 A graph is connected asymptotically almost surely (a.a.s.) if and only if the probability that it is connected tends to one as its number of vertices tends to infinity.

Graph $\mathcal{G}$ is connected a.a.s.

$$
\lim _{n \rightarrow \infty} \mathrm{P}[\mathcal{G} \text { is connected }]=1 \text {. }
$$


Note that for overlay graphs, the vertices are the overlay nodes. This means that $D(n)$ must be such that $\lim _{n \rightarrow \infty} D(n) n=+\infty$.

\section{Problem and model discussion}

Connected basic graph. We consider only connected basic graphs. This seems reasonable to us as a disconnected basic graph will not provide connected overlays, whatever the neighbourhood range is, unless all the overlay nodes are concentrated in a connected part of it.

Asymptotics. Many asymptotic properties of random geometric graphs have been demonstrated [Penrose, 2003]. In particular, we mentioned in Sect. 2 several studies of the asymptotic connectivity of ad hoc networks, while the connectivity probability of a finite network, because of its complexity, has been the subject of very few analytical studies [Desai and Manjunath, 2002].

Our asymptotic results can be seen as approximations of finite (real) networks when the number of nodes is large. They also shed some light on the relation linking $n, r, D$ and $R$ to get a connected overlay.

Network density. Asymptotically, the model presented induces that the overlay nodes geographical density, i.e. the number of overlay nodes per unit area, tends to infinity. This is why it is only suited to so-called dense networks. There exists a more general model, covering dense and sparse networks, that was introduced in [Santi and Blough, 2003], and for which we present similar results in an extended version of this paper [Calomme and Leduc, 2006].

Homogeneous transmission range assignment. The transmission range is represented as a function of the number of nodes. This allows us to model a possible topology control protocol running on the ad hoc network, which would reasonably reduce the transmission range as the number of nodes increases, in order to conserve energy and global network capacity. We however implicitly limit ourselves to homogeneous topology control protocols, i.e. protocols which assign the same transmission range to all nodes.

This assumption greatly simplifies further mathematical developments and seems realistic in the context of our study. A common transmission range at each node provides some appealing features [Kawadia and $\mathrm{Ku}-$ mar, 2005]. Moreover, a common power is asymptotically nearly optimal in terms of network capacity [Narayanaswamy et al., 2002]. 


\section{Mathematical analysis}

\section{Known Results on Basic Graphs}

Consider a basic graph $g(n, r)$. Let us build a graph $g \prime(n, r \prime)$ that has the same nodes set as $g$ and such that there is an edge between every pair of nodes. Let $M_{n}$ denote the longest edge length of the minimal spanning tree built on $g \prime$. In [Penrose, 1997], it is demonstrated that the graph $g(n, r)$ is connected if and only if $r \geq M_{n}$ and

$$
\forall \alpha \in R: \lim _{n \rightarrow+\infty} P\left[n \pi M_{n}^{2}-\ln n \leq \alpha\right]=\exp \left(-e^{-\alpha}\right)
$$

This implies directly the following theorem.

THEOREM 2 (Asymptotic connectivity of basic graphs)

A graph $g(n, r)$ with

$$
\pi r^{2}=\frac{\ln n+k(n)}{n}
$$

is connected a.a.s. if and only if $\lim _{n \rightarrow+\infty} k(n)=+\infty$.

The same result was demonstrated by Gupta and Kumar for a uniform distribution of nodes over the unit disk [Gupta and Kumar, 1999].

\section{Overlay graphs study}

We begin with a theorem that sets an upper bound on the asymptotic number of hops between any pair of nodes, given the distance separating them and the normalized transmission range used.

THEOREM 3 (Asymptotic path length)

Let $g$ be an a.a.s. connected graph and $m$ be a strictly positive integer. Let $n_{1}$ and $n_{2}$ be two nodes of $g$. If the Euclidean distance between $n_{1}$ and $n_{2}$ is strictly less than $m r$, then there exists a.a.s. a path between them composed of less than or exactly $m$ hops.

The detailed demonstrations of this theorem and of the next one are published in the extended version of this paper [Calomme and Leduc, 2006]. We only draw here the sketch of their proof.

Proof 4 (summary) Asymptotic path length

We use a recurrent approach .

If $m=1$, then nodes $n_{1}$ and $n_{2}$ are physical neighbours and the property is valid.

If $m>1$, then it can be demonstrated that there exists a.a.s. a node $n_{i}$ such that the distance between $n_{1}$ and $n_{i}$ is strictly less than $(m-1) r$ and 
that the distance between $n_{i}$ and $n_{2}$ is strictly less than $r$. Consequently, the property is valid for any $m$.

Using the previous theorem, we can derive the main result of this paper.

THEOREM 5 (Asymptotic connectivity of dense overlay graphs)

Consider an overlay graph $G(g, D(n), R(n))$ with

$$
\pi(R r)^{2}=\frac{\ln (\lceil D n\rceil)+K(n)}{\lceil D n\rceil}
$$

Assume $g(n, r(n))$ is a.a.s. connected and $\lim _{n \rightarrow+\infty} D n=+\infty$. $G$ is a.a.s. connected if and only if $\lim _{n \rightarrow+\infty} K(n)=+\infty$.

Proof 6 (summary) Asymptotic connectivity of dense overlay graphs

Let $G(n, r, D, R)$ be an overlay graph.

Consider a graph $g \prime(\lceil D n\rceil, R r)$ such that the vertices sets of $G$ and $g \prime$ are identical.

As the maximal edge length of $G$ equals $R r$, its edges set is included in the edges set of $g /$. If $g^{\prime}$ is not connected, then $G$ neither is.

By definition, any edge $\left(n_{1}, n_{2}\right)$ of $g$ is shorter than or has length $R r$. If it is strictly shorter than $\mathrm{Rr}$, then, by theorem 3, this edge also exists in $G$.

If it has length $R r$, then it can be demonstrated that one can find a node $n_{i}$ such that two edges $\left(n_{1}, n_{i}\right)$ and $\left(n_{i}, n_{2}\right)$, each strictly shorter than $R r$, belong to $G$.

Consequently, if there exists a path between two nodes of $g \prime$, there also exists a path between these nodes in $G$.

If $g$ ' is connected, then $G$ also is.

Applying Theorem 2 to $\mathrm{g}$ ', we obtain a necessary and sufficient condition for the asymptotic connectivity of $G$.

\section{Discussion}

The following two corollaries are meant to give an insight about the relationship between the neighbourhood range and the overlay density. Their proof, quite simple, are given in the extended version of this paper [Calomme and Leduc, 2006].

For both of them, we consider an overlay graph $G(g, D, R)$ and make the assumptions that $g$ is a.a.s. connected and that $\lim _{n \rightarrow+\infty} D n=+\infty$. 
Corollary 7 If $D R^{2} \geq 1$ then $G$ is a.a.s. connected.

The sufficient condition $R>\frac{1}{\sqrt{D}}$ shows that a decreasing overlay density does not necessarily make the overlay graph a.a.s. disconnected. We can for example have $D=\frac{1}{\ln n}$ and $R=\sqrt{\ln n}$. It also confirms the intuitive idea that the lower $D$ is, the larger $R$ must be.

The advantage of the previous corollary is that we do not need any information about the basic graph, except that it is a.a.s. connected. However, lower values for the neighbourhood range could be obtained if the relationship existing between $n$ and $r$ is known.

Corollary 8 Let $\pi r^{2} n=\ln n+k(n)$ with $k(n) \gg 1$. Assume $D$ is constant and $R$ is an integer with $R \geq 1$.

1 If $k(n) \gg \ln n$, then $G$ is a.a.s. connected for any $R$.

2 If $k(n) \geq a \ln n$ with $a>0$, then $G$ is a.a.s connected for any $R>\frac{\frac{1}{\sqrt{D(1+a)}}}{\sqrt{2}}$

3 If $k(n) \ll \ln n$, then $G$ is a.a.s. connected if and only if $R \geq \frac{1}{\sqrt{D}}$.

Concerning a basic graph, a function $k(n)$ that grows quickly just accelerates the convergence of the connectivity probability [Santi and Blough, 2003]. This function has a stronger impact on the neighbourhood range needed for connectivity. For example, for a constant overlay density $D$, it decides if $R$ can take any value or must be greater than a fixed threshold.

In particular, if the transmission range $r$ is kept constant while the number of nodes grows, we have $k(n) \gg \ln n$ which implies that $R=1$ is sufficient to obtain an a.a.s. connected overlay. The overlay nodes do not need other intermediary nodes to forward their packet for communicating. The subnetwork composed of the overlay nodes only is a.a.s. connected. In fact, there is no need for building an overlay in this case; the overlay nodes can directly use their own routing protocol, with customized packet format.

Oppositely, if a topology control protocol is used for optimizing the transmission range, $R=1$ can be too small to make the overlay a.a.s. connected. In this case, the subnetwork composed of the overlay nodes only is a.a.s. disconnected. It is necessary for some overlay nodes to communicate through intermediary non overlay nodes. Overlay techniques are required; the overlay nodes control and data packets must be encapsulated in packets that can be routed by all nodes. 


\section{Conclusions}

We first motivated the study of overlays built over ad hoc networks.

We then presented and analyzed the critical neighbourhood range problem.

In connected networks, as the network gets denser $(n \rightarrow+\infty)$, the shortest path between any pair of nodes draws close to the straight line. This sets an upper bound on the number of hops between any pair of nodes, knowing the distance between them and the nodes normalized radio transmission range $r$.

Thanks to this property, that we called the asymptotic path length theorem, and known work on the critical transmission range problem, one can derive an analytical solution to the critical neighbourhood range problem.

The mathematical condition obtained does take into account the potential use of a homogeneous topology control algorithm and allows the overlay density $D$ to evolve with the number of nodes. In particular, if $D$ diminishes, they show how a compensation in $R$ can keep the overlay still connected.

The analysis of these results provides, among others, the following properties for overlays built on ad hoc networks.

Whatever the characteristics of the underlying network are, an overlay built on an a.a.s. connected network with $D R^{2} \geq 1$ is asymptotically almost surely connected.

In many cases, if the relationship between $n$ and $r$ is known, one can determine a lower value than $\left\lceil\frac{1}{\sqrt{D}}\right\rceil$ for $R$, which will still achieve asymptotic overlay connectivity.

For constant $D$, depending on the network degree of connectivity, the minimal value of $R$ for asymptotic overlay connectivity can either be equal to one, or to a higher fixed threshold, or be an unbounded function of the number of nodes.

In particular, if $D$ and $r$ are kept constant while the number of nodes increases, the overlay nodes can asymptotically use their own routing protocol, bypassing the network routing protocol common to all nodes.

Oppositely, if the transmission range value is optimized, using a topology control protocol for the underlay, the network composed only of the overlay nodes can be a.a.s. disconnected.

\section{Acknowledgments}

This work has been partially supported by the Belgian Science Policy in the framework of the IAP program (MOTION P5/11 project) and by the European Commission (IST E-NEXT NoE). 


\section{References}

Bollobas, B. (1985). Random Graphs. Academic Press, London, England.

Calomme, S. and Leduc, G. (2004). Performance study of an overlay approach to active routing in ad hoc networks. In Proc. of the Third annual Mediterranean Ad Hoc Networking Workshop (Med-Hoc-Net'04), Bodrum, Turkey.

Calomme, S. and Leduc, G. (2006). The critical neighbourhood range for asymptotic overlay connectivity in ad hoc networks. To appear in Ad Hoc and Sensor Wireless Networks.

Desai, M. and Manjunath, D. (2002). On the connectivity in finite ad hoc networks. IEEE Commun. Lett., 10(6):437-490.

Erdos, P. and Rényi, A. (1960). On the evolution of random graphs. Hungarian Academy of Science, 5:17-61.

Goel, A., Rai, S., and Krishnamachari, B. (2004). Sharp thresholds for monotone properties in random geometric graphs. In Proc. of the thirty-sixth annual ACM symposium on Theory of computing (STOC'04), pages 580-586, Chicago, IL.

Gupta, P. and Kumar, P. (1999). Critical power for asymptotic connectivity in wireless networks. In McEneaney, W.M., Yin, G., and Zhang, Q., editors, Stochastic Analysis, Control, Optimization and Applications, A Volume in Honor of W. $H$. Fleming. Birkhäuser, Boston.

Kawadia, V. and Kumar, P.R. (2005). Principles and protocols for power control in wireless ad hoc networks. IEEE J. Select. Areas Commun., 23(1):76-88.

Krishnamachari, B., Wicker, S., and Bejar, R. (2001). Phase transition phenomena in wireless ad-hoc networks. In Proc. IEEE Global Conference on Telecommunications (Globecom'01), Symposium on Ad-Hoc Wireless Networks, San Antonio, TX.

Mohapatra, P., Gui, C., and Li, J. (2004). Group communications in mobile ad hoc networks. IEEE Computer, 37(2):52-59.

Narayanaswamy, S., Kawadia, V., Sreenivas, R.S., and Kumar, P. R. (2002). Power control in ad-hoc networks: Theory, architecture, algorithm and implementation of the compow protocol. In Proc. of European Wireless 2002. Next Generation Wireless Networks: Technologies, Protocols, Services and Applications, pages 156162, Florence, Italy.

Penrose, M. (2003). Random Geometric Graphs. Oxford University Press, England.

Penrose, M. D. (1997). The longest edge of the random minimal spanning tree. The Annals of Applied Probability, 7(2):340-361.

Penrose, M. D. (1999). On the $k$-connectivity for a geometric random graph. Random Structures and Algorithms, 15(2):145-164.

Santi, P. and Blough, D. M. (2003). The critical transmitting range for connectivity in sparse wireless ad hoc networks. IEEE Trans. Mobile Comput., pages 25-39.

Tang, D. and Baker, M. (2000). Analysis of a local-area wireless network. In Proc. of the Sixth Annual International Conference on Mobile Computing and Networking (MOBICOM'00), Boston, MA.

Wang, K. H. and Li, B. (2002). Efficient and guaranteed service coverage in partitionable mobile ad-hoc networks. In Proc. Annual Joint Conference of the IEEE Computer and Communications Societies (INFOCOM'02), New York, NY. 